Instituto Internacional de Investigación y Desarrollo Tecnológico Educativo INDTEC, C.A.

DOI: https://doi.org/10.29394/Scientific.issn.2542-2987.2020.5.E.8.165-184

OAI-PMH: http://www.indteca.com/ojs/index.php/Revista Scientific/oai

Artículo Original / Original Article

\title{
El vínculo figura cuidadora-niño en casos de autismo
}

\author{
Autoras: Silvia de los Ángeles Armas Arboleda \\ Universidad Internacional SEK, UISEK \\ sdarmas.mpp@uisek.edu.ec \\ Quito, Ecuador \\ https://orcid.org/0000-0002-1678-321X \\ Mónica Patricia Cansignia Alcocer \\ Universidad Internacional SEK, UISEK \\ mpcansignia.mpp@uisek.edu.ec \\ Quito, Ecuador \\ https://orcid.org/0000-0001-9311-1217 \\ Elena Narcisa Díaz Mosquera \\ Universidad Internacional SEK, UISEK \\ endiaz@puce.edu.ec \\ Quito, Ecuador \\ https://orcid.org/0000-0002-6093-2614
}

\section{Resumen}

El espectro autista (TEA) es un trastorno del neurodesarrollo caracterizado por dificultades en socialización, empatía, comunicación verbal y no verbal, conductas e intereses repetitivos y estereotipados. Si bien en todos los niños es fundamental la presencia de figuras cuidadoras sensibles a sus necesidades, esta labor se dificulta en los niños con TEA por las particularidades del trastorno. Por tanto, el objetivo del presente estudio fue explorar las características de la interacción de cuatro figuras cuidadoras con sus niños con TEA para proveer recomendaciones que fortalezcan este vínculo, de modo que se promueva el desarrollo de los niños. Se utilizó una metodología de enfoque cualitativo y de tipo exploratorio y descriptivo. Las herramientas empleadas fueron una ficha de datos sociodemográficos y el test MBPQS para obtener una apreciación de la sensibilidad de las figuras cuidadoras participantes en el cuidado de sus niños con TEA. Los resultados demostraron que había manifestaciones del cuidado sensible que requerían ser fortalecidas. A partir de estos hallazgos se elaboraron recomendaciones a las figuras cuidadoras, con pautas para fortalecer el vínculo y el desarrollo de los niños.

Palabras clave: niño; desarrollo del niño; madre; padre; papel de los padres.

Cómo citar este artículo:

Armas, S., Cansignia, M., \& Díaz, E. (2020). El vínculo figura cuidadora-niño en casos de autismo. Revista Scientific, 5(Ed. Esp.), 165-184, e-ISSN: 2542-2987. Recuperado de: https://doi.org/10.29394/Scientific.issn.2542-2987.2020.5.E.8.165-184

Fecha de Recepción: 09-04-2020
Fecha de Aceptación: 22-06-2020
Fecha de Publicación: 05-08-2020 
OAI-PMH: http://www.indteca.com/ojs/index.php/Revista_Scientific/oai

Artículo Original / Original Article

\title{
The caregiver-child bond in autism cases
}

\begin{abstract}
Autistic spectrum (ASD) is a neurodevelopmental disorder characterized by difficulties in socialization, empathy, verbal and nonverbal communication, repetitive and stereotyped behaviors and interests. Although the presence of caregivers who are sensitive to their needs is essential in all children, this task is difficult in children with ASD due to the particularities of the disorder. Therefore, the objective of the present study was to explore the characteristics of the interaction of four caregivers with their children with ASD in order to provide recommendations that strengthen this bond, so as to promote the development of children. An exploratory and descriptive qualitative approach methodology was used. The tools used were a sociodemographic data sheet and the MBPQS test to obtain an appreciation of the sensitivity of the participating caregivers in the care of their children with ASD. The results showed that there were manifestations of sensitive care that needed to be strengthened. Based on these findings, recommendations were made to caregivers, with guidelines to strengthen the bond and development of children.
\end{abstract}

Keywords: children; child development; mother; father; parent role.

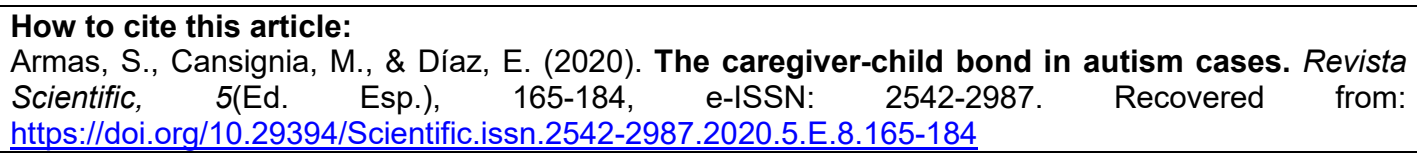

Date Received:

09-04-2020
Date Acceptance:

22-06-2020
Date Publication:

05-08-2020 


\section{Introducción}

Haciendo un recorrido histórico sobre el origen de la palabra autismo, Alcantud y Alonso (2015a): mencionan que el término, introducido por Eugen Bleuler (1857-1939) en el año 1908, en sus estudios sobre la demencia precoz, se deriva del griego autos que se refiere a lo propio, a uno mismo, además mencionan, que posteriormente fue utilizado por Leo Kanner (18941981) en el año 1943, para referirse a once niños que presentaban un desarrollo diferente en cuanto al lenguaje, la comunicación, el desarrollo cognitivo y social. También Alcantud y Alonso (2015b): refieren que en el año 1944, Hans Asperger (1906-1980) utilizó el término psicopatología autista en relación con la sintomatología encontrada en cuatro niños que presentaron síntomas similares a los descritos por Kanner, pero sin retraso en el lenguaje, por lo que en el año 1981, Lorna Wing (1928-2014) le llama Síndrome de Asperger a la psicopatología autista, en honor a su descubridor.

La Organización Mundial de la Salud, World Health Organization (2001): presentó una tipificación multiaxial de los trastornos psiquiátricos en niños y adolescentes, dentro de la Clasificación Internacional de Enfermedades, versión 10 (ClE-10), con el fin de detectar signos de autismo. En este documento se enlistó al Autismo infantil con una codificación (F84.0), el cual fue caracterizado por desarrollo atípico desde antes de los tres años, alteraciones en la interacción social recíproca y en la comunicación y por formas restrictivas, repetitivas y estereotipadas de comportamiento, intereses y actividades. Actualmente, el Ministerio de Salud Pública del Ecuador (2017): utiliza esta clasificación para el diagnóstico de autismo.

La Asociación Estadounidense de Psiquiatría, American Psychiatric Association (APA, 2014a): presenta la quinta edición del Manual Diagnóstico y Estadístico de los Trastornos Mentales (DSM-5). En este manual, el autismo aparece como trastorno del espectro autista (TEA), entidad que reúne a varias categorías en las que se presentan dificultades en la interacción social, entre 
ellas el Síndrome de Asperger. En el DSM-5, el TEA es definido como un trastorno del neurodesarrollo que se caracteriza, esencialmente, por dificultades en interacción social, comunicación verbal y no verbal, y por la presencia de conductas repetitivas y estereotipadas. Para la American Psychiatric Association (APA, 2014b): estas conductas pueden presentarse en un rango de 1 a 3 , según el grado de deterioro y la necesidad de ayuda requerida.

En cuanto a la prevalencia, aunque el DSM-5, de acuerdo con la American Psychiatric Association (APA, 2014c): indica que un promedio internacional de $1 \%$ de la población presenta esta condición, como lo indica Maenner, et al. (2020): se ha reportado una tasa en aumento, pues se ha identificado la presencia de autismo en 1 de cada 54 niños. López y Larrea (2017a): no obstante, en América Latina se ha advertido una ausencia de estudios confiables de prevalencia debido a la dificultad en el diagnóstico y a la escasez de investigaciones.

En el caso del Ecuador, Un estudio publicado por Dekkers, Groot, Díaz, Andrade y Delfos (2015): buscó establecer la prevalencia de niños con autismo en la educación regular en la ciudad de Quito, Ecuador, para lo cual se levantó información en 161 instituciones educativas que albergaban un total de 51,463 estudiantes; se encontró que había apenas un $0.11 \%$ de niños y jóvenes con diagnóstico de autismo y un $0.21 \%$ con sospecha de TEA, matriculados en educación regular.

Posteriormente, López y Larrea (2017b): en un estudio realizado en el año 2017 con 160 niños (50\% neurotípicos y 50\% con diagnóstico previo de autismo) provenientes de varias ciudades de la Costa y de la Sierra ecuatoriana, encontró que 69 de los 80 niños diagnosticados con TEA presentaban rasgos de autismo, mientras que en los 11 restantes se podía descartar la presencia del trastorno. Estos estudios apoyan la noción de una escasez de diagnósticos certeros de TEA en el Ecuador. 
Adicionalmente, en los 69 niños ecuatorianos que presentaban autismo en el estudio de López y Larrea (2017c): las autoras identificaron una carencia de respuestas a las caricias de afecto con una frecuencia 6,7 veces mayor en comparación con los niños neurotípicos. En referencia al factor de interacción social, Ruggieri (2014a): ha explicado que los niños con autismo presentan expresiones faciales diferentes y que tienen dificultades para reconocer las expresiones de los demás, aun cuando se trate de sus cuidadores o de personas muy cercanas, debido a la carencia de lectura de los ojos. Esta situación les imposibilita percibir gestos, emociones, deducir intenciones, comprender miradas, deseos y entender las conductas del otro, lo cual afecta el comportamiento social y la empatía.

Interpretando a Flórez (2000): se entiende por figura cuidadora a aquella persona que asume el rol de proporcionar apoyo y cubrir las necesidades de alguien que no está en condiciones de cuidarse a sí mismo. El cuidado es una actividad formal e informal, pues puede ser ejercida por un profesional de enfermería o por la familia y allegados. En un cuidado efectivo, lo más destacable es el proceso de interacción que se presenta entre persona cuidadora y persona cuidada. Esta relación se ve enriquecida por los momentos de cuidado, las tareas y las actividades compartidas y la constancia en el cuidado ofrecido; estos factores inciden en el desarrollo de un vínculo entre ambas personas.

En el caso de los niños con TEA, afirma Rojas (2017): que las figuras cuidadoras están sujetas a los cambios que pueden dar sus vidas, ya que deben adaptarse a las necesidades de los niños, lo que podría incluir sobrellevar dificultades a nivel emocional y social, además de estar expuestas a la crítica por las creencias erróneas o mitos que estigmatizan a las personas con autismo y a su familia en general. Al cubrir afectivamente las necesidades especiales de los niños con TEA, las figuras cuidadoras fomentan su desarrollo y contribuyen a mejorar la calidad de vida y la interacción social de los 
menores.

En referencia a este tema, es esencial mencionar que Ainsworth (1973), citada por Salinas-Quiroz y Posada (2015): planteó la noción de sensibilidad del cuidador como un modo de describir la habilidad del padre, madre o cuidador/a de acoger las señales del niño, interpretarlas y responder adecuadamente. Es decir, una figura sensible prestará atención no solo a las necesidades físicas del niño, sino también a sus necesidades emocionales y sociales. Además, la figura cuidadora sincroniza sus actividades con las del niño, concierta con él ante la presencia de problemas, se percata de los momentos emocionales por los cuales está atravesando el niño y por las particularidades de su etapa del desarrollo.

Por tanto, se ha planteado que la seguridad del apego es producto de la sensibilidad de la figura cuidadora. La seguridad del apego se define para Bowlby (1988a): como la confianza que experimenta el niño en que su figura cuidadora o figura de apego, está accesible y disponible para brindarle protección o consuelo si una necesidad aparece. Este vínculo primario con los cuidadores constituye la base del desarrollo socio emocional del niño y determina en gran medida, la calidad de sus relaciones futuras con las demás personas.

En consecuencia, Bowlby (1988b): expone que el establecimiento del vínculo figura cuidadora-niño, los dos integrantes de la díada tienen un rol fundamental. En un vínculo saludable, a través de la atención y cuidado sensible, la figura cuidadora le transmite al niño que puede sentirse seguro con ella y confiar en su disponibilidad. El niño, gracias al desarrollo neuronal que progresivamente alcanza, capta este mensaje de la figura cuidadora, establece un vínculo seguro con ella y aprende él también a mostrarse empático y sensible hacia los demás. Por lo tanto, para Briceño (2018): la empatía es un componente esencial en el desarrollo óptimo de las relaciones de apego y en las interacciones sociales, pues permite entender y dar sentido 
a las acciones que tiene un individuo hacia otro y comprender los estados mentales de los demás.

Señalando a Ruggieri (2014b): en el niño con autismo se presenta un déficit en cuanto a la comprensión de las emociones de los otros y en cuanto al desarrollo de empatía. La baja reactividad de su mirada le dificulta el reconocimiento facial y la detección de expresiones emocionales, lo cual le genera una disfunción en la socialización, imitación y desarrollo de la motricidad. Debido a ello, el curso regular del establecimiento de un vínculo afectivo, primero con los cuidadores y luego con las demás personas, suele verse alterado, lo cual afecta aún más, el desarrollo socio afectivo del niño con TEA.

Varias investigaciones a nivel internacional han explorado la relación que se presenta entre figura cuidadora-niño con autismo y la calidad del vínculo afectivo que se genera entre ellos el cual, como lo mencionan Kahane y El-Tahir (2015): recibe la influencia de varios factores, entre ellos las particularidades del TEA en el niño y las manifestaciones de la sensibilidad de su figura cuidadora. De allí, que nos preguntamos: ¿De qué manera se podría fortalecer el vínculo figura cuidadora-niño con autismo?

Para buscar la respuesta a esta pregunta, el objetivo del presente estudio fue explorar las manifestaciones de la sensibilidad de las figuras cuidadoras de cuatro niños con autismo, con la finalidad de proveer recomendaciones para fomentar el desarrollo de los niños, a través de aproximaciones y respuestas más efectivas por parte de las figuras cuidadoras. El fin último es contribuir al desarrollo, en el niño con TEA, de un vínculo basado en confianza y seguridad.

\section{Metodología}

El presente estudio fue llevado a cabo con un enfoque cualitativo de tipo transversal pues se analizaron los hechos observados en la relación figura 
cuidadora-niño con autismo en un solo espacio de tiempo, y se interpretaron los resultados, lo que permitió encontrar y determinar significados. Es un estudio de tipo exploratorio y descriptivo, ya que se buscó explorar y describir las características del vínculo en la díada para generar recomendaciones que fortalezcan estas relaciones. Todos los procedimientos empleados fueron previamente aprobados por el Comité de Bioética de la Universidad San Francisco de Quito (USFQ), Ecuador.

\subsection{Participantes}

Participaron en el presente estudio cuatro figuras cuidadoras de niños que tenían un diagnóstico de autismo, seleccionadas de manera no probabilística y de forma intencional y decisional.

Las cuatro participantes eran de sexo femenino, madres y cuidadoras principales de los niños con TEA, quienes, al momento del estudio, acudían a un grupo de consultorios médicos y psicológicos que atiende población con trastornos del desarrollo. Las edades de las participantes estaban comprendidas entre 26 y 44 años. Cada una de ellas tenía a su cargo un hijo/a con autismo. Los niños (una mujer y tres hombres) tenían entre 5 y 10 años.

\subsection{Instrumentos}

Como instrumentos se utilizó una ficha sociodemográfica elaborada por las investigadoras y la escala de Posada, Kaloustian, Richmond y Moreno (2007a): del Comportamiento materno para preescolares Q-set, Maternal Behavior for Preschoolers Q-set (MBPQS), adaptada a las expresiones lingüísticas utilizadas en el Ecuador, recabando información relevante, concerniente tanto a datos de identificación, como a los antecedentes, diagnóstico y tratamiento de los niños.

Con la escala MBPQS se obtuvo una apreciación de la disposición de las figuras cuidadoras en la atención a los niños con TEA. Según Posada, 
Kaloustian, Richmond y Moreno (2007b): esta escala puede ser aplicada mediante observación de las interacciones habituales de la díada en su ambiente natural, o mediante auto-reporte de la figura cuidadora, que fue la modalidad que se empleó en el presente estudio.

De manera previa a la aplicación del instrumento, los ítems del MBPQS fueron transformados a preguntas, para ser administrados a cada figura cuidadora a modo de entrevista. Las respuestas obtenidas, fueron evaluadas con la técnica Q-sort propia del instrumento, la cual, de acuerdo a Posada, Kaloustian, Richmond y Moreno (2007c): consiste en que los 90 ítems, escritos en tarjetas individuales, se dividen, en primera instancia, en tres categorías de conductas: muy características, ni características ni no características y no características. En segunda instancia, cada una de estas tres categorías se dividen en tres subcategorías con 10 ítems en cada una, con lo cual se obtiene nueve grupos con tarjetas que van de conductas muy características (grupo 9) a conductas no características en absoluto (grupo 1). Cada ítem recibe un puntaje en un rango de 9 a 1 , en función del grupo en el que fue ubicado.

\subsection{Procedimiento}

Las figuras participantes fueron reclutadas a través del grupo de consultorios médicos y psicológicos a los cuales acuden por la atención de sus hijos con TEA. Una vez seleccionadas, se las invitó a participar en el estudio, para lo cual, en una reunión individual, se les dio toda la información necesaria y se suscribió con ellas el documento de consentimiento informado. En un segundo encuentro se les aplicó la ficha sociodemográfica y el cuestionario elaborado con el MBPQS. En un encuentro final se compartieron recomendaciones para fortalecer el vínculo con sus hijos con TEA.

Los puntajes asignados a las figuras cuidadoras en la aplicación del MBPQS, fueron contrastados con los puntajes del criterio normativo de sensibilidad del instrumento. Se identificaron los ítems en los que las 
respuestas de las figuras cuidadoras presentaban las discrepancias más altas (<3 puntos) con los puntajes del criterio de sensibilidad, y sobre aquella base se elaboró la guía.

\section{Resultados}

Como resultado de la aplicación de la ficha sociodemográfica, las figuras cuidadoras reportaron tener o haber tenido algún familiar con Síndrome de Asperger, síndrome de Down y epilepsia. Reportaron también, que durante el embarazo del niño con TEA presentaron condiciones de salud tales como anemia, preclamsia, problemas de tiroides. En cuanto a redes de apoyo, manifestaron recibir la ayuda de abuelos, tíos, hijos mayores.

Respecto de los resultados de la aplicación del MBPQS, se registraron nueve (9) conductas que se presentaban fortalecidas en las figuras cuidadoras, en la que una corresponde a las dimensiones de la sensibilidad de Contribución a Interacciones Armoniosas (Cl). Las otras ocho (8) conductas no forman parte de las dimensiones, pues son parte de los ítems relacionados con emocionalidad y sociabilidad. En la tabla 1, se describen estas conductas y se las presenta con el ítem del MBPQS al que hacen referencia.

Tabla 1. Conductas fortalecidas en las figuras cuidadoras.

\begin{tabular}{|c|c|l|}
\hline Dimensión & $\begin{array}{c}\text { Ítem al } \\
\text { que hace } \\
\text { referencia }\end{array}$ & \multicolumn{1}{|c|}{ Descripción de la conducta } \\
\hline \multirow{2}{*}{$\mathrm{Cl}$} & 29 & $\begin{array}{l}\text { En la comunicación con el niño utilizan palabras, gestos } \\
\text { o miradas, haciendo un importante uso de lenguaje } \\
\text { verbal y no verbal. }\end{array}$ \\
\hline \multirow{4}{*}{$\begin{array}{c}\text { Otras } \\
\text { conductas }\end{array}$} & 1 & $\begin{array}{l}\text { Las figuras cuidadoras se dan cuenta cuando sus } \\
\text { niños/as sonríen y vocalizan. }\end{array}$ \\
\cline { 2 - 3 } & 18 & $\begin{array}{l}\text { Proveen a los niños un ambiente físico adaptado a sus } \\
\text { necesidades. }\end{array}$ \\
\cline { 2 - 3 } & 20 & $\begin{array}{l}\text { Animan y buscan alternativas para que sus niños } \\
\text { interactúen con sus pares. }\end{array}$ \\
\cline { 2 - 3 } & 26 & $\begin{array}{l}\text { Evitan términos peyorativos y no minimizan a los niños } \\
\text { cuando los describen. }\end{array}$ \\
\cline { 2 - 3 } & 32 & Intentan proporcionar juguetes apropiados para la edad \\
\hline
\end{tabular}




\begin{tabular}{|c|l|l|}
\hline & \multicolumn{1}{|c|}{ y su condición de TEA. } \\
\cline { 2 - 3 } & 41 & $\begin{array}{l}\text { Cuando salen de casa, llevan los implementos } \\
\text { necesarios para su niño (ropa, alimentos, abrigo o } \\
\text { pañal). }\end{array}$ \\
\cline { 2 - 3 } & $\begin{array}{l}\text { Cuidan la apariencia física de sus niños intentando } \\
\text { mantenerlos limpios y aseados. }\end{array}$ \\
\hline \multirow{2}{*}{82} & $\begin{array}{l}\text { Enseñan a sus hijos a usar el lenguaje utilizando } \\
\text { expresiones cortas, como, por ejemplo: Arriba "Upa", Sí, } \\
\text { No, entre otras. }\end{array}$ \\
\hline
\end{tabular}

Fuente: Las Autoras (2020).

Se identificaron también veintiún (21) conductas que presentaban altas discrepancias ( $<3$ puntos) con los puntajes del criterio normativo de sensibilidad. De ellas, dos (2) corresponden a la dimensión de Contribución a Interacciones Armoniosas ( $\mathrm{Cl})$, seis (6) a la dimensión de Apoyo de Base Segura (ABS), tres (3) a Supervisión y Monitoreo (SUP), y tres (3) a Establecimiento de Límites (EL). Las siete (7) conductas restantes no pertenecen a las dimensiones de la sensibilidad, pues forman parte del grupo de ítems relacionados con estado de ánimo, emocionalidad, sociabilidad. En la tabla 2, se presenta la descripción de estas conductas, así como el ítem del MBPQS con el que se relacionan.

Tabla 2. Conductas que presentan discrepancias con el criterio normativo de sensibilidad.

\begin{tabular}{|c|c|c|}
\hline Dimensión & $\begin{array}{l}\text { Ítem al } \\
\text { que hace } \\
\text { referencia }\end{array}$ & Descripción de la conducta \\
\hline \multirow[b]{2}{*}{$\mathrm{Cl}$} & 28 & Control e intrusión en las interacciones con el niño. \\
\hline & 60 & $\begin{array}{l}\text { Posición crítica, parece fastidiada o expresa palabras } \\
\text { ofensivas. }\end{array}$ \\
\hline \multirow{6}{*}{ ABS } & 5 & Interacciones a distancia. \\
\hline & 36 & No se toman en cuenta los intereses del niño. \\
\hline & 51 & Falta de interés o afecto cuando el niño regresa. \\
\hline & 55 & $\begin{array}{l}\text { Respuesta inadecuada cuando ocurre un accidente con } \\
\text { el niño. }\end{array}$ \\
\hline & 56 & Demora su respuesta cuando el niño llora. \\
\hline & 59 & $\begin{array}{l}\text { No intenta reasegurar al niño cuando éste llora o emite } \\
\text { señales. }\end{array}$ \\
\hline
\end{tabular}


Instituto Internacional de Investigación y Desarrollo Tecnológico Educativo INDTEC, C.A.

DOI: https://doi.org/10.29394/Scientific.issn.2542-2987.2020.5.E.8.165-184

OAI-PMH: http://www.indteca.com/ojs/index.php/Revista_Scientific/oai

Artículo Original / Original Article

\begin{tabular}{|c|c|c|}
\hline \multirow{3}{*}{ SUP } & 40 & No evita situaciones conflictivas para el niño. \\
\hline & 72 & Se distrae frecuentemente con otras demandas. \\
\hline & 74 & $\begin{array}{l}\text { Interviene en las actividades del niño incluso cuando no } \\
\text { es necesario. }\end{array}$ \\
\hline \multirow{3}{*}{ EL } & 67 & Enuncia las reglas al niño sin explicaciones. \\
\hline & 70 & $\begin{array}{l}\text { Severidad excesiva ante el comportamiento arriesgado } \\
\text { o peligroso. }\end{array}$ \\
\hline & 84 & $\begin{array}{l}\text { Permite que el niño se desorganice a causa de sus } \\
\text { estados emocionales. }\end{array}$ \\
\hline \multirow{7}{*}{$\begin{array}{l}\text { Otras } \\
\text { conductas }\end{array}$} & 14 & Regaña al niño. \\
\hline & 19 & $\begin{array}{l}\text { Percibe el comportamiento del niño como una forma de } \\
\text { rechazo hacia el cuidador/a. }\end{array}$ \\
\hline & 50 & No permite al niño apartarse a una distancia segura. \\
\hline & 69 & Parece abrumada por las solicitaciones del niño. \\
\hline & 79 & $\begin{array}{l}\text { Intenta detener la expresión de sentimientos por parte } \\
\text { del niño. }\end{array}$ \\
\hline & 83 & Abandona la habitación sin dar explicaciones al niño. \\
\hline & 89 & $\begin{array}{l}\text { Pasa por alto señales y oportunidades para interactuar } \\
\text { con el niño. }\end{array}$ \\
\hline
\end{tabular}

Fuente: Las Autoras (2020).

De manera adicional, las figuras cuidadoras manifestaron, durante la entrevista de aplicación del MBPQS, que las conductas de sus niños con TEA a veces las desalientan y no saben cómo actuar, por lo que en ocasiones responden con comportamientos intimidantes o intrusivos.

\section{Conclusiones}

El autismo es un trastorno que cada vez se presenta con mayor incidencia y que al ser difícil de diagnosticar, plantea una dificultad a la hora de conocer cifras exactas sobre su prevalencia. Esta situación obstaculiza la atención y tratamiento oportuno del niño, así como la sensibilización adecuada, no solo de su círculo familiar, sino también del contexto en el que se desenvuelve, en donde muchas veces las personas tienen un desconocimiento del autismo y de sus manifestaciones.

El Ecuador no está exento de esta realidad y aunque en muchos de los documentos oficiales del país se reconocen los derechos de las personas con 
autismo y sus familias, poco es lo que se ha hecho a nivel práctico para garantizarles la provisión de un ambiente seguro y protegido, en el que se fomente permanentemente el desarrollo de vínculos sólidos con las personas a cargo de su cuidado y atención. Bajo esta perspectiva, el objetivo del presente estudio fue explorar las manifestaciones de la sensibilidad de las figuras cuidadoras de un pequeño grupo de niños con autismo, con la finalidad de proveer recomendaciones para fomentar el desarrollo de los niños, a través de aproximaciones y respuestas más efectivas por parte de los cuidadores.

Los resultados demostraron que una de las conductas fortalecidas en el cuidado sensible de los niños con autismo pertenece a la dimensión de contribución a interacciones armoniosas, y se refiere al hecho de que, en general, las figuras cuidadoras utilizan palabras, gestos, miradas en la comunicación con sus hijos con TEA, haciendo un importante uso de lenguaje verbal y no verbal cuando interactúan con ellos. Además, las figuras cuidadoras reportaron estar atentas a las señales de comunicación de sus niños, proveerles un ambiente físico adaptado a sus necesidades, con juguetes apropiados para su edad y condición de TEA. También reportaron estar pendientes de su apariencia física y aseo, llevar los implementos necesarios para sus hijos cuando salen de casa, animarlos a interactuar con otros niños, enseñarles el uso de expresiones comunicativas cortas, evitar el uso de términos peyorativos y subvalorativos cuando los describen.

Sin embargo, también se identificaron conductas que requieren ser fortalecidas en las figuras cuidadoras. Por ejemplo, ante la dificultad de manejar las conductas de sus hijos, a veces ellas se muestran excesivamente controladoras e incluso reorientan físicamente a los niños, pues por momentos llegan a sentirse exhaustas con las continuas demandas de cuidado que a causa del trastorno se presentan. Esta situación las lleva a que en ocasiones mantengan cierta distancia con los niños, se muestren poco afectuosas con ellos, se demoren en responder a sus señales, provean reglas estrictas sin 
explicaciones, actúen con severidad. Por otro lado, la conducta de los niños y su escasez de respuesta emocional, a veces ocasiona que las figuras cuidadoras se sientan rechazadas por ellos o que tomen su mal comportamiento como algo personal.

Frente a estos hallazgos, se elaboraron recomendaciones de actividades que puedan ser llevadas a cabo por las figuras cuidadoras, con el respaldo y supervisión del psicólogo tratante. El propósito fue sensibilizar a las figuras cuidadoras y fortalecer el vínculo entre ellas y los niños con autismo, de tal forma que se resignifiquen las respuestas que estas figuras les proporcionan, para que los niños perciban un ambiente seguro y confiable que contribuya a su desarrollo y al establecimiento de nuevas conductas comunicativas y de funcionamiento simbólico.

Es importante tener en mente que los niños con autismo presentan un retraso en su desarrollo social. A pesar de tener edades cronológicamente más avanzadas, en muchos casos su desarrollo socio afectivo se ha estancado dentro de la primera infancia, razón por la cual es fundamental fomentar el desarrollo de conductas saludables de apego y de vínculos afectivos seguros, labor en la cual las figuras cuidadoras tienen un papel esencial. Varios estudios y planteamientos teóricos afirman que el niño con TEA puede establecer un vínculo de apego seguro con sus figuras cuidadoras en etapas más avanzadas que los niños típicos, siempre que estas figuras se muestren sensibles en el cuidado que les otorgan.

Es importante anotar que el auto-reporte de las manifestaciones conductuales que las figuras cuidadoras presentan ocasionalmente y a las que se ha hecho referencia, ponen en evidencia la necesidad de un proceso de acompañamiento que las ayude a sostenerse. Aunque cuentan con redes de apoyo dentro de sus familias para la atención de los niños, requieren de programas de cuidado al cuidador, tema que ha sido abordado por varias investigaciones internacionales, con la finalidad de que se sientan fortalecidas 
para desempeñar su demandante y complejo rol.

Consecuentemente, se recomienda el empoderamiento no solo de las figuras cuidadoras primarias, sino también de las redes de apoyo que se constituyen en figuras cuidadoras secundarias y que pueden brindar a los niños un acompañamiento de base segura. Se debe recordar que el papel de la familia en los procesos educativos de los niños es esencial.

\section{Referencias}

Alcantud, F., \& Alonso, Y. (2015a,b). Trastornos del Espectro Autista. En Moreno Osella, E (Ed) Necesidades Educativas Especiales: Una mirada diferente. ISBN: 978-84-606-6197-9. Córdoba, España: Fedune.

American Psychiatric Association (2014a,b,c). DSM-5. Manual Diagnóstico y Estadístico de los Trastornos Mentales. 5ta Edición, ISBN: 9788491101727. Buenos Aires, Argentina: Editorial Médica Panamericana.

Bowlby, J. (1988a,b). Una base segura. Buenos Aires, Argentina: Ediciones Paidós.

Briceño, V. (2018). Trastornos del Espectro Autista en Educación Inicial: Experiencia Educativa. Revista Scientific, 3(8), 218-233, e-ISSN: 2542-2987. Recuperado de:

https://doi.org/10.29394/Scientific.issn.2542-2987.2018.3.8.11.218-233 Dekkers, L., Groot, N., Díaz, E., Andrade, I., \& Delfos, M. (2015). Prevalence of Autism Spectrum Disorders in Ecuador: A Pilot Study in Quito. Journal of Autism and Developmental Disorders, 45, 4165-4173, eISSN: 1573-3432. Recovered from: https://doi.org/10.1007/s10803-015$\underline{2559-6}$

Flórez, J. (2000). El síndrome del cuidador. Jano: Medicina y humanidades, (1345), 46-50, ISSN: 0210-220X. España: Doyma.

Kahane, L., \& El-Tahir, M. (2015). Attachment behavior in children with 
Autistic Spectrum Disorders. Advances in Mental Health and Intellectual Disabilities, 9(2), 79-89, e-ISSN: 2044-1282. Recovered from: https://doi.org/10.1108/AMHID-06-2014-0026

López, C., \& Larrea, M. (2017a,b,c). Autismo en Ecuador: un Grupo Social en Espera de Atención. Revista Ecuatoriana de Neurología, 26(3), 203-214, e-ISSN: 1019-8113. Recuperado de:

http://revecuatneurol.com/magazine issue article/autismo-ecuadorgrupo-social-espera-atencion-autism-ecuador-social-group-waitingattention/

Maenner, M., Shaw, K., Baio, J., Washington, A., Patrick, M., DiRienzo, M., ... Dietz, P. (2020). Prevalence of Autism Spectrum Disorder Among Children Aged 8 Years - Autism and Developmental Disabilities Monitoring Network, 11 Sites, United States, 2016. MMWR: Surveillance Summaries, 69(SS-4), 1-12, e-ISSN: 1545-8636. Recovered from: http://dx.doi.org/10.15585/mmwr.ss6904a1

Ministerio de Salud Pública del Ecuador (2017). Trastornos del Espectro Autista en niños y adolescentes: detección, diagnóstico, tratamiento, rehabilitación y seguimiento. Quito: Ministerio de Salud Pública, Dirección Nacional de Normatización-MSP.

Posada, G., Kaloustian, G., Richmond, M., \& Moreno, A. (2007a,b,c). Maternal secure base support and preschoolers' secure base behavior in natural environments. Attachment \& Human Development, 9(4), 393411, e-ISSN: 1461-6734. Recovered from:

https://doi.org/10.1080/14616730701712316

Rojas, A. (2017). Creencias erróneas y cumplimiento del cuidador en padres de niños con autismo de educación especial de Lima Sur. Tesis. Lima, Perú: Universidad Autónoma del Perú. Recuperado de: http://repositorio.autonoma.edu.pe/bitstream/AUTONOMA/367/1/ROJA S\%2OHUAYTA\%2C\%20ANDREA\%20LUCERO.pdf 
Ruggieri, V. (2014a,b). La amígdala y su relación con el autismo, los trastornos conductuales y otros trastornos del neurodesarrollo. Revista de Neurología, 58(Supl. 1), S137-S148, e-ISSN: 1576-6578. Recuperado de: https://doi.org/10.33588/rn.58S01.2013571

Salinas-Quiroz, F., \& Posada, G. (2015). MBQS: Método de evaluación para intervenciones en apego dirigidas a primera infancia. Revista Latinoamericana de Ciencias Sociales, Niñez y Juventud, 13(2), 10511063, e-ISSN: 2027-7679. Recuperado de:

http://revistaumanizales.cinde.org.co/rlcsnj/index.php/Revista-

Latinoamericana/article/view/1986

World Health Organization (2001). Clasificación multiaxial de los trastornos psiquiátricos en niños y adolescentes: clasificación de la CIE-10 de los trastornos mentales y del comportamiento en niños y adolescentes. ISBN: 8479034912. Madrid, España: Editorial Panamericana. 
OAI-PMH: http://www.indteca.com/ojs/index.php/Revista_Scientific/oai

Artículo Original / Original Article

\section{Silvia de los Ángeles Armas Arboleda}

e-mail: sdarmas.mpp@uisek.edu.ec

Nacida en Quito, Ecuador, el 25 de julio del año 1978.

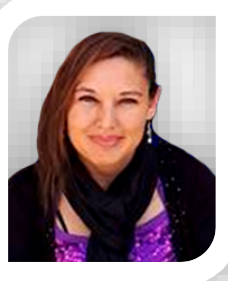

Magíster en Psicología con mención en Psicoterapia, Psicóloga Infantil y Psicorehabilitadora; Licenciada en Ciencias de la Educación, especialización Parvularia; me he desempeñado como docente de educación parvularia; directora académica; psicóloga infantil y 


\section{Mónica Patricia Cansignia Alcocer \\ e-mail: mpcansignia.mpp@uisek.edu.ec}

Nacida en Sangolquí, Ecuador, el 17/ de diciembre del

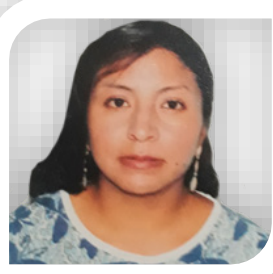
año 1978. Magíster en Psicología con mención en Psicoterapia; Psicóloga General; me he desempeñado como psicóloga en instituciones educativas y en proyectos psicosociales; y como docente de apoyo a las necesidades educativas especiales; ejecutora de talleres y seminarios sobre violencia intrafamiliar. 
OAI-PMH: http://www.indteca.com/ojs/index.php/Revista_Scientific/oai

Artículo Original / Original Article

\section{Elena Narcisa Díaz Mosquera}

e-mail: endiaz@puce.edu.ec

Nacida en Esmeraldas, Ecuador, el 22 de agosto del año

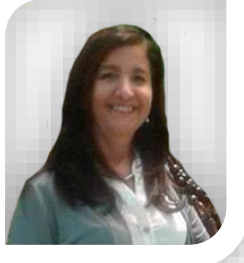
1959. Doctora en Psicología; Magíster en Educación; Licenciada en Psicología con especialización en Psicología Clínica; Docente de educación superior, atención en consulta psicológica; mis Líneas de investigación son: relaciones vinculares, autismo, educación; tengo varias publicaciones sobre los temas mencionados.

El contenido de este manuscrito se difunde bajo una Licencia de Creative Commons ReconocimientoNoComercial-Compartirlgual 4.0 Internacional 